\title{
The Construction of Intelligent English Teaching Model Based on Artificial Intelligence
}

\author{
https://doi.org/10.3991/ijet.v12.i12.7963 \\ Xiaoguang Li \\ Taiyuan University, Taiyuan, Shanxi, China \\ lixiaoguangivan@ $126 . \mathrm{com}$
}

\begin{abstract}
In order to build a modernized tool platform that can help students improve their English learning efficiency according to their mastery of knowledge and personality, this paper develops an online intelligent English learning system that uses Java and artificial intelligence language Prolog as the software system. This system is a creative reflection of the thoughts of expert system in artificial intelligence. Established on the Struts Spring Hibernate lightweight JavaEE framework, the system modules are coupled with each other in a much lower degree, which is convenient to future function extension. Combined with the idea of expert system in artificial intelligence, the system developed appropriate learning strategies to help students double the learning effect with half the effort; Finally, the system takes into account the forgetting curve of memory, on which basis the knowledge that has been learned will be tested periodically, intending to spare students' efforts to do a sea of exercises and obtain better learning results.
\end{abstract}

Keywords - Artificial intelligence, foreign language teaching, intelligent learning

\section{$1 \quad$ Introduction}

For a long time, the traditional way of teaching English has been widely criticized for various problems and deficiencies, and the crisis often means turning point. With the advancement of the integration of information technology and English curriculum, artificial intelligence also serves as a tool for the optimization of secondary school English teaching. [1] provides a new platform for the creation of an intelligent and personalized English teaching environment.

In this paper, a teaching aid system is designed based on artificial intelligence and the forgetting law of human brains [2]. This system can review English vocabulary and knowledge points lest they are forgotten over time, so as to improve learning efficiency and provide targeted learning contents [3]. Through practical research, the system is proved with certain application value and referential meanings for the methods alike. 


\section{System design}

The new online English learning system is founded on the JavaEE-based B / S model "as stated in [4]", allowing users in all geographical scopes to visit and log in to the system through a browser. The system development is a tri-layer JavaEE architecture: the user layer, which is the browser; the web layer, which sends request to transfer data and compute business logics; and the database layer, which completes the reading and writing of data and transfers data to the business logic layer where the information is processed into webpage displayable ones and sent back to the browser for users to view [5]. The system uses Windows XP Professional as the operational system, Java1.6 development language, Eclipse + MyEclipse 8 project development integrated environment, and Dreamweaver 8 static page development environment.

\subsection{System overall structure}

Combined with the SSH framework, the system's function and performance are analyzed and shown in Figure 1.

The whole system is based on the SSH framework and its role is mainly to solve the decoupling problem, to strengthen the connection between simple structures, to make the hierarchy between each structure clear, to provide a quick way to develop code, and to increase the scalability and maintainability of the system. "As stated in [6] ". The system is composed of student self-learning system and an expert system that assists college English teaching. There are four user types in total, i.e. administrators, students, English teachers and experts. They can use different privileges under the auto-control of the system.

1. Administrator, which maintains the system in a regular time basis. His job is to change the password, manage the query, addition, modification and deletion of test bases and experts, and manage student users and teacher users.

2. Experts, referring to the senior English teachers with years of teaching experience who can provide sample templates necessary for neural networks and who can analyze and modify the knowledge points and difficulty of papers in the test bases. Their major work is to maintain the query, deletion, modification and addition of neural network systems and static/dynamic knowledge bases.

3. Students, who can input the user name and password to log into the system, do exercises and self-tests, and take target training according to the test results and the principle of memory. 
Paper-The Construction of Intelligent English Teaching Model Based on Artificial Intelligence

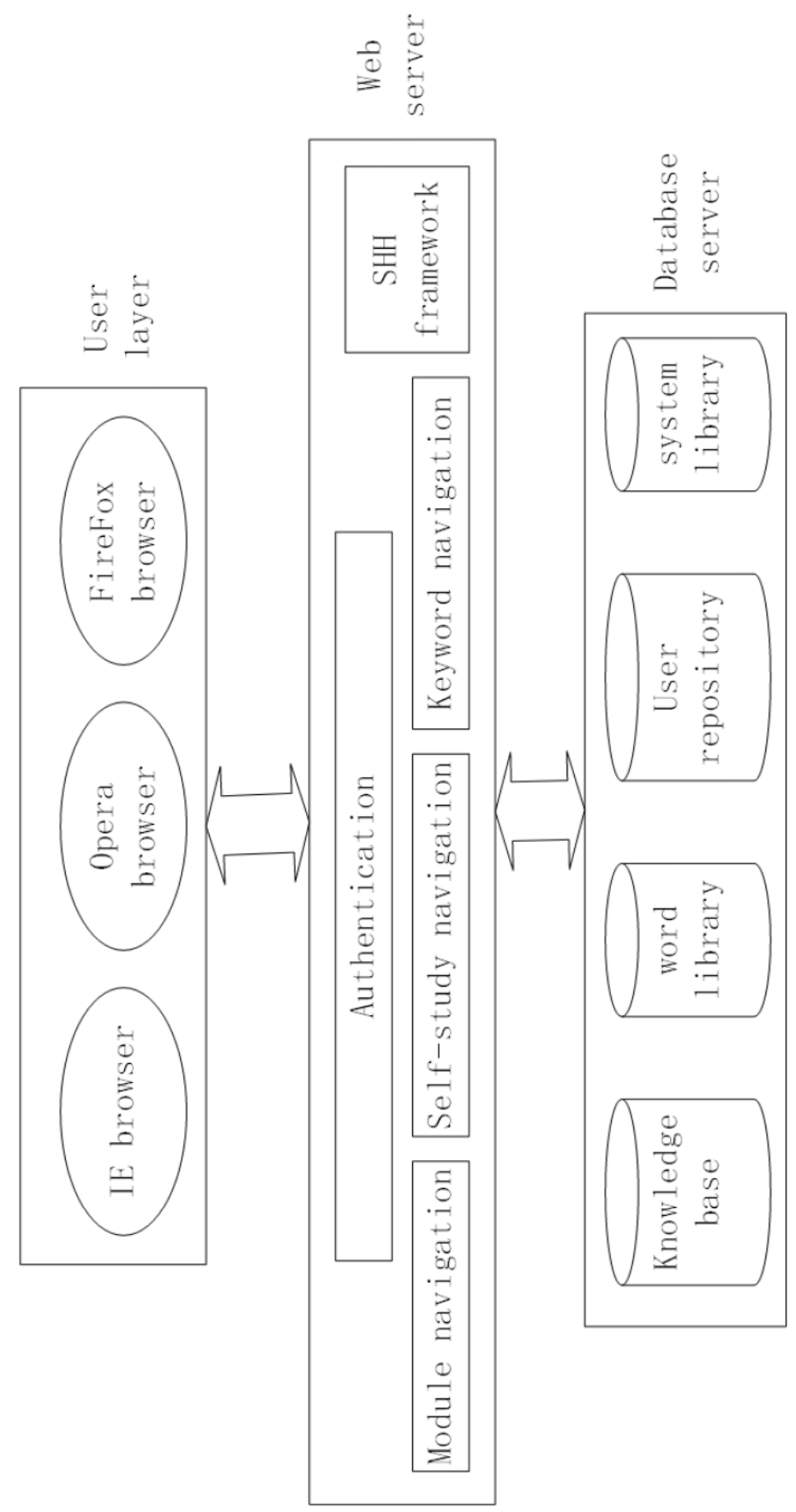

Fig. 1. The overall framework of online English learning system 


\subsection{Expert system knowledge base design}

Knowledge representation refers to the thresholds and weights distributed in the network, rather than an independent rule. There are differences of the knowledge representation system between neural network knowledge base and traditional expert system. The neural network repository uses an implicit representation and inherent mechanism "as stated in [7]." Neurons can output and store the information of threshold and weight, and establish knowledge base to determine the neural network. The establishment of knowledge base is mainly about the establishment of two processes of storage and acquisition, which is also an online learning process. Expert network construction is to establish a knowledge base to provide learners with knowledge.

The knowledge acquisition path is to modify the weight of self-adaptive algorithms and learn the training samples provided from the expert system, until reaching the learning goal. Then, the heuristic questions will be released on the Internet. There are many nodes on the path of knowledge acquisition, each of which has the corresponding neuron to connect the weight and the input information. When meeting the knowledge that needs to be stored, the neuron will automatically distribute them in every threshold and connection weight. The network intrusion detection accuracy (G) is used as the objective function of neural network parameter optimization, and thus the optimization problem of RBF neural network parameters can be described as:

$$
\begin{aligned}
& \max _{M} G(M) \\
& M=\left\{w_{i j}, c_{i}, \sigma_{i}\right\} \\
& w_{i j} \geqslant 0 \\
& c_{i} \geqslant 0 \\
& \sigma_{i} \geqslant 0
\end{aligned}
$$

$w_{i j}$ denotes the connection strength from the ith neuron to the jth neuron, ci denotes the central value of the implicit function, and $\sigma \mathrm{i}$ denotes the kernel width.

\subsection{Knowledge point intelligent module design}

Figure 2 shows the model of student knowledge structure. The student cognitive module consists of two modules: learning state and learning tracking. The main function is to judge students' record contents, knowledge level and data contents. The model mines and collects student knowledge levels through three channels, implicit information, explicit information, and background information "as stated in [9]." Implicit information is obtained during problem solving and student answers; explicit information is obtained when students use the system; and background information is mainly derived from the student's level. 


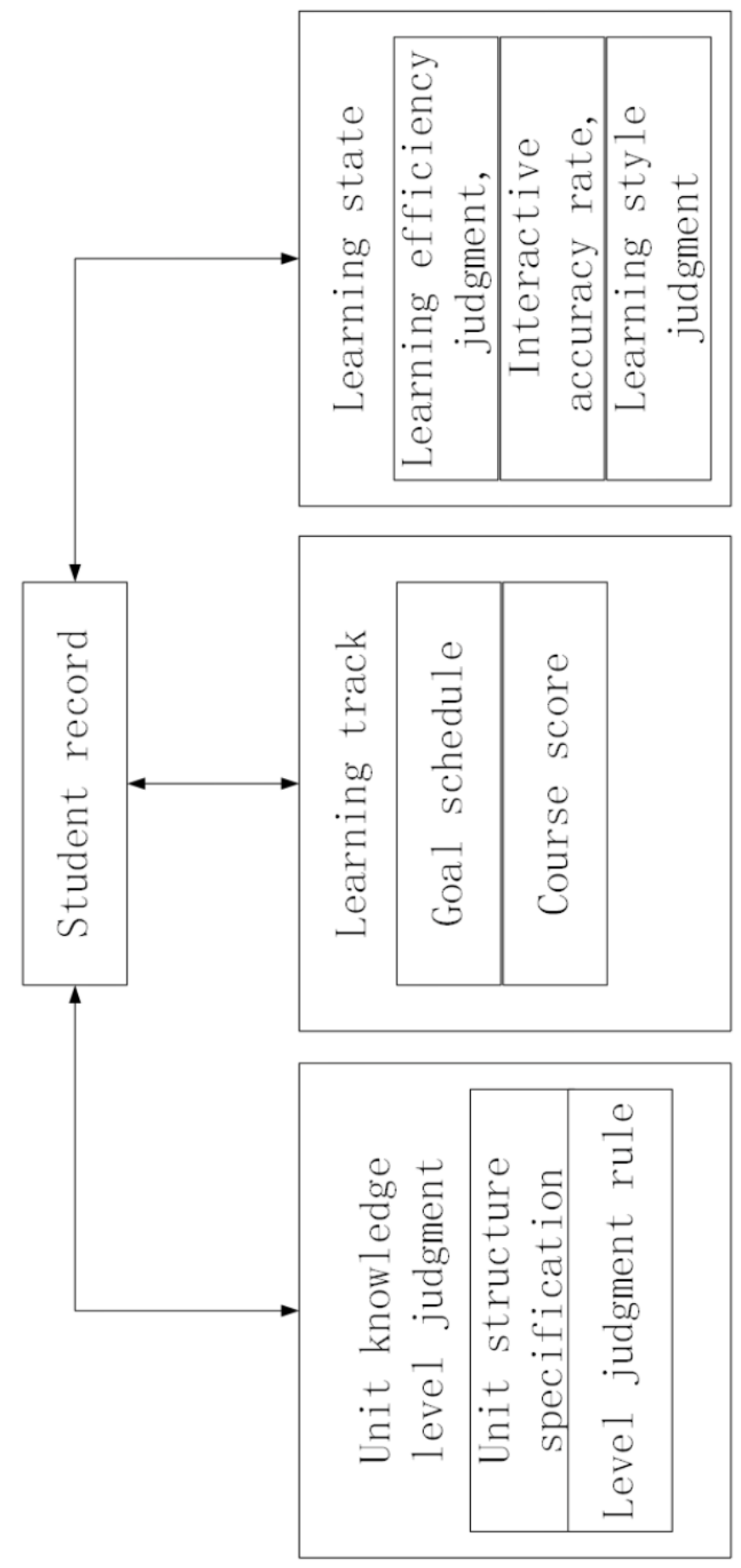

Fig. 2. Student knowledge structure model 
Authorware is a file manipulation function. When a student first logs in to the system, WriteExtFile will create a record based on the account password and other information filled in by the student login system, provided that the initial value of each item is zero. In addition, the student's record number is stored in the record as a variable. If the student wants to logs in again, there will be no need to enter the relevant information. Instead, the student record number in the data item or query column will be called and sent to Presentuser.

\subsection{Knowledge point display module}

The neural network expert system can calculate which knowledge points need to be grasped, remembered again, or strengthened according to the previous level of mastery of knowledge. In addition to browsing knowledge points, users can search for them in the search column through knowledge name, specialized vocabulary, and source materials. Then, the key words are inputted, and the user clicks the search button. If there is no search result, the system will give a prompt of "no user information found", and send messages to the administrator to add or update information. Search results, if any, will be displayed to the user. The key to user query is to identify query conditions and show the information to the user. The flow charts of the algorithm and key word navigation are shown in Figure 3 and Figure 4, respectively.

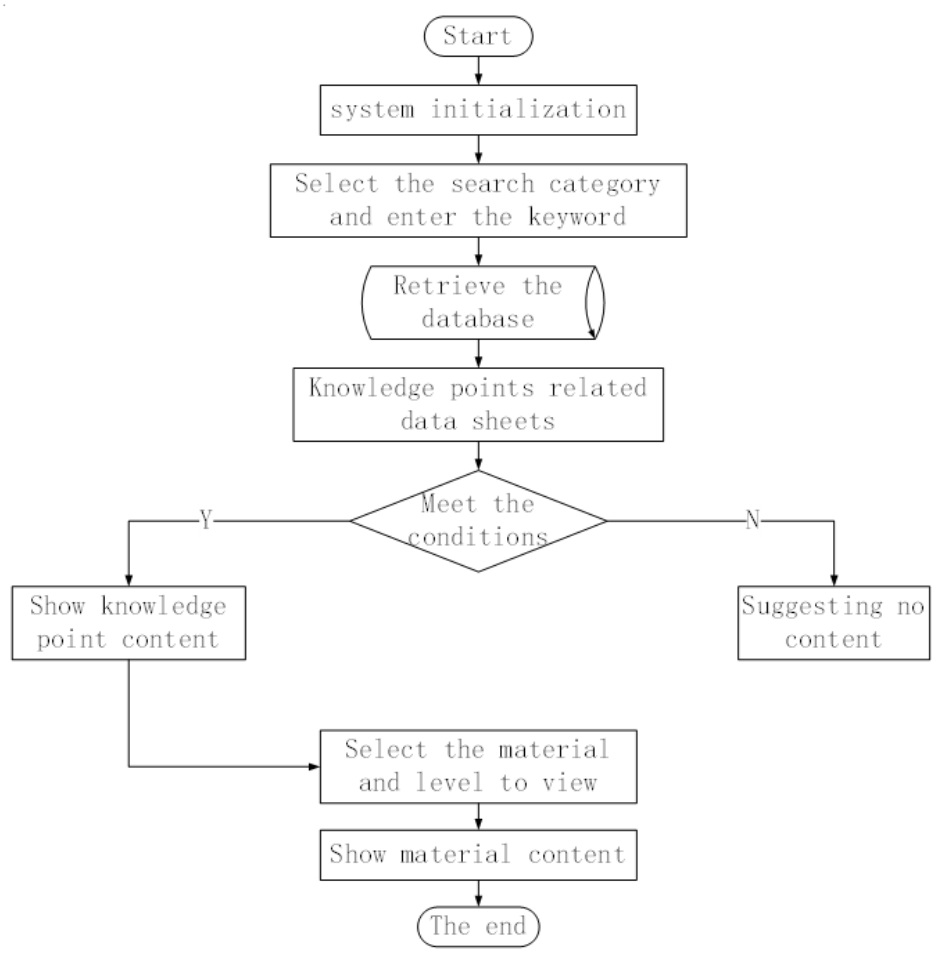

Fig. 3. User query knowledge point algorithm 


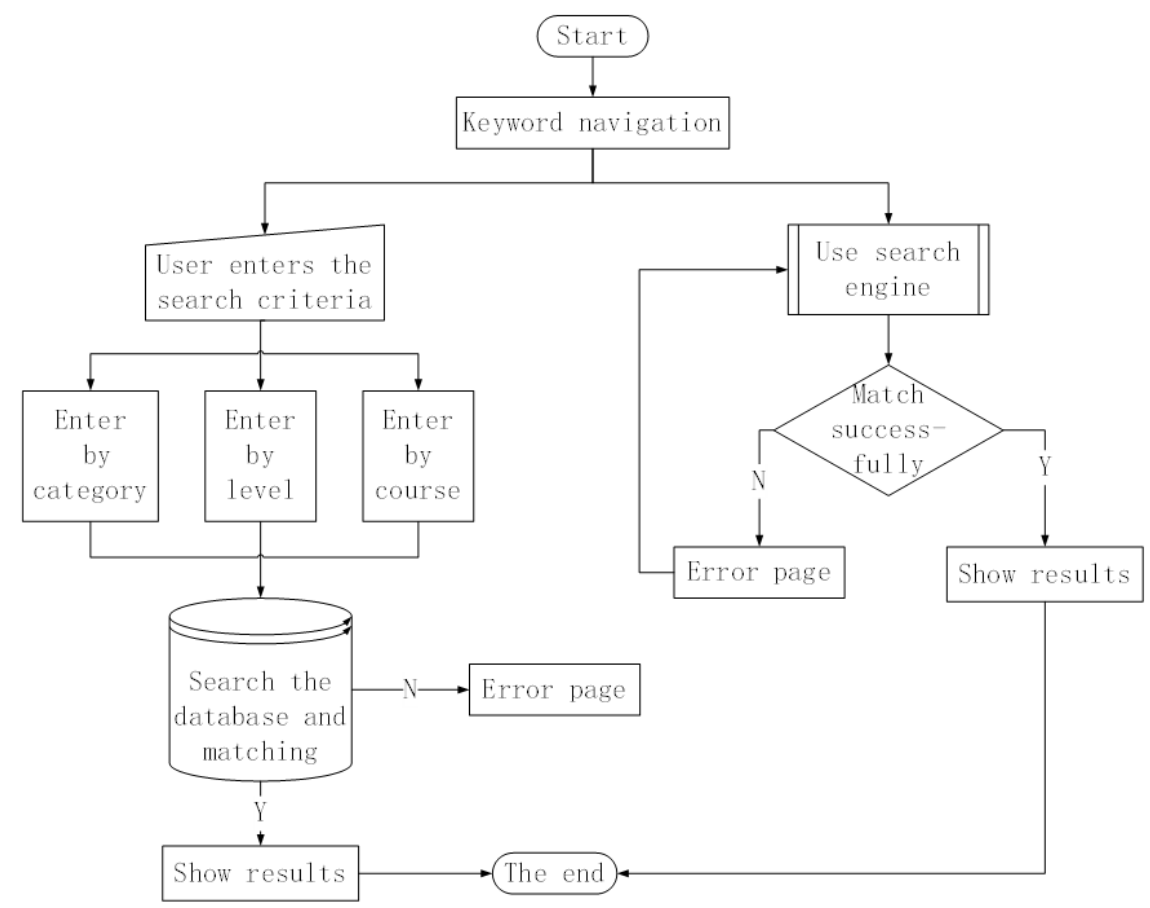

Fig. 4. The steps of algorithm deletion and modification are similar to those of algorithm addition. Keyword navigation

\subsection{Background management module}

In the vocabulary query page is the way to search vocabulary so that users can quickly find the expected information. The vocabulary is added and deleted by the administrator. The specific algorithm is shown in Figure 5.

\section{System test results and discussion}

The pressure test software WebCT is used for system test, which enables the server and client to work normally as stated in [10]. The data results displayed on the client's page reflect the system's ability to meet the customer requirements in the early stage of the design process.

Six interface tests are conducted and the results are as follows:

1. Button layout on the window should coordinate with the interface, neither too dense nor too sparse, and the result can be passed.

2. Fonts should be uniform (usually Times New Roman), the font size should be moderate, and the results in multiple pages should be the same. 


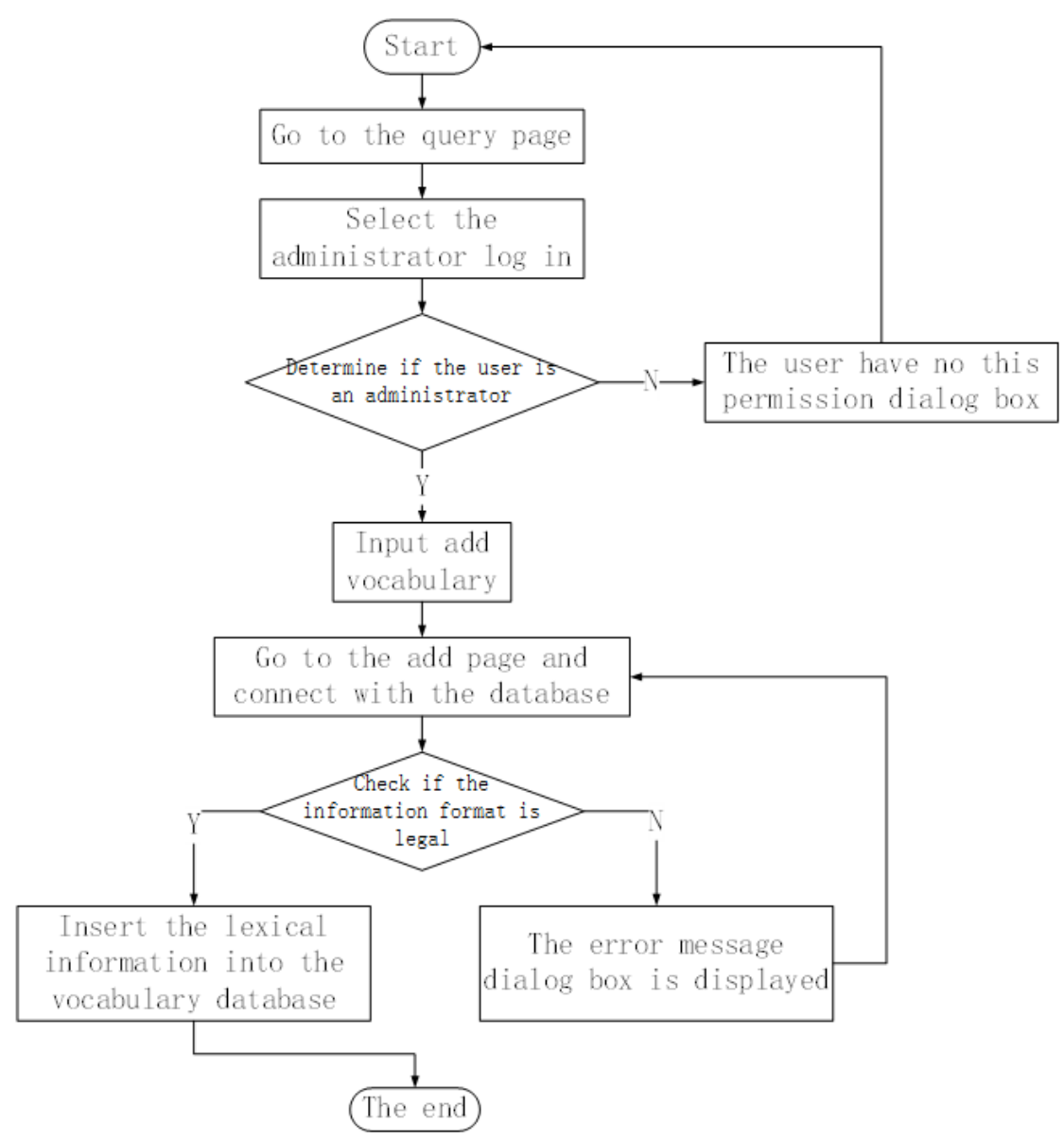

Fig. 5. The addition algorithm of background management module

3. The color matching of the interface should be suitable rather than too bright, and the results can be self-customized.

4. The menu layer should be equal to or less than 3 , the shortcuts do not repeat, and the results show no abnormality.

5. No typos, no mixture of Chinese and English, with accurate names and no abnormal results.

6. Uniform fonts, no mix of full-width and half-width fonts, and the results pass.

We also conduct the performance test and concurrency test, whose details are:

1. Performance test: The test content is the user / administrator login time. First, use loadrunner to simulate 200 users logging in to the system at the same time, lasting 20 minutes; then, do the same simulation except that the simulation time is 10 minutes; finally, use loadrunner to simulate 200 users submitting data at the same time for 20 minutes. The maximum, minimum and average time are recorded. 
2. Concurrency test: The test content is whether the database supports the synchronous operation of massive users. First, use loadrunner to simulate 200 users logging in to the system at the same time for 20 times; then, use loadrunner to simulate 200 users submitting different data at the same time for 20 times; finally, use loadrunner to simulate 200 users submitting the same data at the same time for 20 times. And we verify if the system can support these operations.

Experimental results find 2 serious defects, 14 general defects, and 21 minor defects. This outcome means that the interface is basically stable and usable, but there should be some improvements.

\section{Conclusion}

With the SSH framework as the basis, we design and implement the intelligent English learning system. The knowledge of expert systems in artificial intelligence is creatively applied to English knowledge point memory and learning process, during which knowledge points are manifested as a multi-attribute variable. The neural network algorithm is used in the learning process when the forgetting law of human brain is considered in target training. Referring to the common methods and procedures of software test, we test the existing hardware and software systems until they achieve the overall goal. In the follow-up study, the authors will conduct in-depth and optimized research on the system, so that the system involves a wider coverage and richer contents.

\section{$5 \quad$ References}

[1] Konstantin, E. B. Mikhail, A. N. Boris, A. Kruze. (2014). The International Teacher's Foreign Language Professional Communicative Competency Development. Procedia - Social and Behavioral Sciences, 154: 329-332. https://doi.org/10.1016/j.sbspro.2014.10.158

[2] Antar, A. Abdelbaset H. (2017). Medieval Muslim thinkers on foreign language pedagogy: The case of Ibn Khaldun. Lingua, 193: 62-71. https://doi.org/10.1016/j.lingua.2017.05.001

[3] Hao-Chiang, K. L. Ching-Ju, C. Tsu-Ching, H. (2015). From a perspective on foreign language learning anxiety to develop an affective tutoring system. Educational Technology Research and Development, 63(5): 727-747. https://doi.org/10.1007/s11423-015-9385-6

[4] Chun, L. (2015). Modeling teachers' influence on learners' self-directed use of technology for language learning outside the classroom. Computers \& Education, 82: 74-83. https://doi.org/10.1016/j.compedu.2014.11.005

[5] Valentina, K. (2015). Developing Foreign Language Teaching: Regional Experience. Procedia - Social and Behavioral Sciences, 214: 614-618. https://doi.org/10.1016/j.sbs pro.2015.11.767

[6] Qingmei, L. (2012). Single-polaron properties in the one-dimensional Holstein and SSH models. Physics Letters A, 376(14): 1219-1225. https://doi.org/10.1016/j.physleta. 2012.02.026

[7] Xiaoming, Z. Bo, L. Qiaosheng, G. (2015). Construction of a haustorium development associated SSH library in Thesium chinense and analysis of specific ESTs included by Imperata cylindrica. Biochemical Systematics and Ecology, 64: 46-52. 
[8] Yongchang, R. Deyi, J. Tao, X. (2011). Research on software development platform based on SSH framework structure. Procedia Engineering, 15: 3078-3082. https://doi.org/10.1016/j.proeng.2011.08.577

[9] Paulo, M. Fernandes, J. M. Luisa, B. (2013). Evaluating the feasibility of GPS measurements of SSH on board a ship along the Portuguese West Coast. Advances in Space Research, 51(8): 1492-1501. https://doi.org/10.1016/j.asr.2012.10.028

[10] Sathyabhama, M. Viswanathan, R. Nandakumar, M. (2015). Understanding sugarcane defence responses during the initial phase of Colletotrichum falcatum pathogenesis by suppression subtractive hybridization (SSH). Physiological and Molecular Plant Pathology, 91: 131-140. https://doi.org/10.1016/j.pmpp.2015.07.003

\section{$6 \quad$ Author}

Xiaoguang Li is with Taiyuan University, Taiyuan, Shanxi, China.

Article submitted 29 October 2017. Published as resubmitted by the author 08 December 2017. 